\title{
An Approach to Empirical Time Series Analysis
}

\author{
Emanuel Parzen \\ Department of Statistics, Stanford University, Stanford, Calif.
}

(Received December 6, 1963; revised January 24, 1964)

\begin{abstract}
This paper attempts to develop a philosophy for empirical time series analysis, involving the routine use of four data handling procedures (covariance estimation, spectral estimation, autoregressive model fitting and spectral estimation, and trend elimination and estimation) embodied in a computer program.

The cross-spectral analysis of a pair of time series, each consisting of 4000 observations, requires approximately 10 minutes on a 7090, including computation of covariances. Several examples of empirical time series analysis are given.
\end{abstract}

\section{Introduction}

The probabilistic theory of time series is now extensively developed, built on the pioneering work of Wiener, Khintchine, Kolmogorov, Cramér, Loève, and Karhunen in the 1930's and 1940's. The statistical theory also enjoys an extensive literature and a fair share of beautiful results. While the probabilistic theory can be pursued for the sake of its great beauty, it would be a mistake if the statistical theory were to be developed only for its elegance. The ultimate aim of the statistical theory must be to provide data handling procedures for achieving the aim of time series analysis: synthesis of stochastic models which can be used to describe, and perhaps to control, the mechanisms generating each time series and relating various time series.

For this reason, one may define a field, which may be called empirical time series analysis, with aims such as the following:

(i) to develop efficient computer programs for the statistical treatment of empirical time series, paying especial attention to flexibility of input and output,

(ii) to develop a philosophy, based on statistical theory, for judging and interpreting the statistical data reduction provided by the computer output,

(iii) to provide experience in the small sample applicability of statistical procedures derived from asymptotic theory,

(iv) to focus attention on the theoretical questions requiring further investigation, such as the problem of how to transform observed data to put them into a form where they satisfy the assumptions required to apply various statistical data reduction routines.

1 Paper presented at the Symposium on Signal Statistics in Seattle, Wash., Dec. 6-7, 1963, under the title "Statistical Methods for Stochastic Processes; Prepared under the auspices of National Science Foundation Grant GP-82.
The goal of empirical time analysis is precisely that enunciated by Richard Hamming [1962] for numerical analysis: the seeking of insight rather than numbers. One should not expect empirical time series analysis to be a mere matter of grinding out answers. Rather one desires to obtain a wealth of answers which can be tempered by imagination and judgement to achieve models for time series.

My aim in this paper is to sketch an approach to empirical time series analysis whose basic attitude is: one should analyze the data in a number of ways, each corresponding essentially to a different possible model for the observed time series. Each analysis provides estimates of the parameters (or incompletely specified probability law characteristics) involved in the model. Comparing the analyses provides rough tests of hypotheses concerning which model provides a better fit to the data.

\section{Standard Models}

The standard model adopted for the analysis of a time series $\left\{X_{i}(t), t=1,2, \ldots\right\}$ is to write it as the sum

$$
X_{i}(t)=m_{i}(t)+Y_{i}(t)
$$

of its mean value function

$$
m_{i}(t)=E\left[X_{i}(t)\right]
$$

and the residuals or fluctuations

$$
Y_{i}(t)=X_{i}(t)-m_{i}(t) .
$$

In order to have the possibility of statistical inference from a single finite sample of a time series, one assumes that the residuals are covariance sta- 
tionary [Parzen, 1962] in the sense that (for $i, j=1,2$ ) there exist functions $R_{i j}(v)$ of integers $v=0, \pm 1, \ldots$. such that

$$
\operatorname{Cov}\left[X_{i}(t), X_{j}(t+v)\right]=E\left[Y_{i}(t) Y_{j}(t+v)\right]=R_{i j}(v) .
$$

We call $R_{i j}(v)$ the covariance functions of the time series, and

$$
\rho_{i j}(v)=\frac{R_{i j}(v)}{\left\{R_{i i}(0) R_{j j}(0)\right\}^{1 / 2}}
$$

the correlation functions.

The covariance functions $R_{i j}(v)$ always possess representations as Fourier-Stieltjes integrals. It is next assumed that they are Fourier transforms of spectral density functions $f_{i j}(\omega)$ :

$$
R_{i j}(v)=\int_{-\pi}^{\pi} e^{\mathrm{i} v \omega} f_{i j}(\omega) d \omega, \quad \mathbf{i}=\sqrt{-1} .
$$

The (auto) spectral density function $f_{i i}(\omega)$ is a nonnegative even function of $\omega$ :

$$
f_{i i}(-\omega)=f_{i i}(\omega) \geq 0, \quad|\omega| \leq \pi .
$$

The cross-spectral density function $f_{i j}(\omega)$ is in general complex valued; its real and imaginary parts are called respectively the co-spectral density, denoted $c_{i j}(\omega)$, and quadrature spectral density, denoted $q_{i j}(\omega)$. These functions possess the following properties (writing $\bar{z}$ to denote the complex conjugate of $z$ )

$$
\begin{aligned}
& f_{i j}(-\omega)=\overline{f_{i j}(\omega)}=f_{j i}(\omega), \\
& c_{i j}(-\omega)=c_{i j}(\omega), \\
& q_{i j}(-\omega)=-q_{i j}(\omega) .
\end{aligned}
$$

One can define for two jointly covariance stationary time series $X_{i}(\cdot)$ a variety of spectral quantities, as follows:

$$
w_{i j}(\omega)=\frac{\left|f_{i j}(\omega)\right|^{2}}{f_{i i}(\omega) f_{j j}(\omega)}=\frac{c_{i j}^{2}(\omega)+q_{i j}^{2}(\omega)}{f_{i i}(\omega) f_{j j}(\omega)}
$$

called the coherency between $X_{i}(\cdot)$ and $X_{j}(\cdot)$ at frequency $\omega$;

$$
g_{i \mid j}(\omega)=\frac{\left|f_{i j}(\omega)\right|}{f_{j}(\omega)}
$$

called the gain at frequency $\omega$ of the predictor of $X_{i}(\cdot)$ given $X_{j}(\cdot)$;

$$
g_{j \mid i}(\omega)=\frac{\left|f_{i j}(\omega)\right|}{f_{i}(\omega)}
$$

called the gain at frequency $\omega$ of the predictor of $X_{j}(\cdot)$ given $X_{i}(\cdot)$;

$$
\varphi(\omega)=\operatorname{arc} \tan \left(\frac{q_{i j}(\omega)}{c_{i j}(\omega)}\right),
$$

called the phase difference between the two series at frequency $\omega$;

$$
f_{j \mid i}(\omega)=f_{j j}(\omega)\left\{1-W_{i j}(\omega)\right\},
$$

called the error spectrum of the predictor of $X_{j}($. given $X_{i}(\cdot)$.

In the sequel we shall discuss the question of estimating the mean value functions and spectral functions associated with time series. In terms of the spectrum, one can devise various physical mechanisms (especially îlters) which might have generated the time series and which might be used to simulate them.

\section{Sample Covariance and Correlation Functions}

A basic step in empirical time series analysis is to form estimates of the covariance, correlation, and spectral functions. I believe it correct to say that these estimation problems do not as yet have generally accepted solutions. One of the aims of this paper is to stress the points at issue.

The first point I desire to raise is that for the sake of developing a modular computer program we should adopt the following definitions for the sample covariance functions $R_{11}(\cdot)$ and $R_{22}(\cdot)$, and sample cross-covariance functions $R_{12}(\cdot)$ and $R_{21}(\cdot)$ : for $i, j=1,2$,

$$
R_{i j}(v)=\frac{1}{N} \sum_{t=1}^{N-|v|} X_{i}(t) X_{j}(t+v), \quad v=0,1, \ldots, N-1 .
$$

For negative values of $v$ we deine

$$
R_{i j}(-v)=R_{i j}(v) \text {. }
$$

For the sake of distinguishing the estimates from the population quantities they are estimating one should write $R_{i j}^{*}(v)$ instead of $R_{i j}(v)$ to indicate that it is an estimate. For ease of writing we omit such asterisks. However, they should be inserted by the reader when discussing the properties of the estimates.

Computing the sample covariance and crosscovariance functions will be one of the most time consuming aspects of a time series analysis of real data. It turns out that one can choose a convenient number $V_{\max }$, less than $N$, such that one need compute only

$$
R_{i j}(v), \quad v=0,1, \ldots, V_{\max } .
$$

One rarely chooses $V_{\max }$ to be greater than 40 percent of the sample size $N$, and often it will be $0.25 \mathrm{~N}$. Some considerations on how to choose $V_{\max }$ are discussed below. We call $V_{\max }$ the covariance truncation point. 
From the sample covariance and cross-covariance functions, one forms sample correlation and crosscorrelation functions, deîned by

$$
\rho_{i j}(v)=R_{i j}(v) /\left\{R_{i i}(0) R_{j j}(0)\right\}^{1 / 2}
$$

for $v=0,1, \ldots, \quad V_{\max }$. As an example of the meaning of (3.3) note that

$$
\rho_{i i}(v)=\frac{\sum_{t=1}^{N-v} X_{i}(t) X_{i}(t+v)}{\sum_{t=1}^{N} X_{i}^{2}(t)}, \quad v=0,1, \ldots, N-1 .
$$

It should be noted that some writers would estimate $\rho_{i j}(v)$ by the ordinary correlation coefficient between the two series

$$
\left\{X_{i}(t), t=1,2, \ldots, N-v\right\} \text { and }
$$

$$
\left\{X_{j}(t+v), t=1,2, \ldots, N-v\right\}
$$

which is given by

$$
\frac{\sum_{t=1}^{N-v}\left(X_{i}(t)-X_{i, 0}^{*}\right)\left(X_{j}(t+v)-X_{i, v}^{*}\right)}{\left\{\sum_{t=1}^{N-v}\left(X_{i}(t)-X_{i}^{*}\right)^{2} \sum_{i=1}^{N-v}\left(X_{j}(t+v)-X_{i, v}^{*}\right)^{2}\right\}^{1 / 2}}
$$

defining

$$
\begin{aligned}
& X_{i, 0}^{*}=\frac{1}{N-v} \sum_{t=1}^{N-v} X_{i}(t), \\
& X_{i, v}^{*}=\frac{1}{N-v} \sum_{t=v+1}^{N} X_{j}(t) .
\end{aligned}
$$

The next two sections discuss the motivations for and implications of definitions (3.1) and (3.3), and why we oppose deinnition (3.6).

\section{Mean Subtraction}

It seems odd that we do not use in the sample covariance function the deviations $X(t)-\bar{X}$ of the observations from the sample mean

$$
\bar{X}=\frac{1}{N} \sum_{t=1}^{N} X(t)
$$

rather than $X(t)$ itself. To explain our position on this point we introduce the notion of detrending.

In analyzing a time series $\{X(t), t=1,2, \ldots, N$, one usually adopts a model for $X(\cdot)$ of the form,

$$
X(t)=m(t)+\epsilon(t),
$$

where $m($.$) is the mean value function, sometimes$ called the trend, and $\epsilon(\cdot)$ is the fluctuation or residual series about the trend assumed to be covariance stationary.

One is interested in the trend for two purposes:

(i) to estimate it, as an important part of the model one is fitting to the time series,

(ii) to eliminate it in such a way as to obtain estimated values of the residual series $\epsilon(\cdot)$ so that its covariance and spectral structure can be estimated.

The optimum procedure for estimating the trend depends on the covariance structure of the residuals. Consequently, in a sense, the problem of trend elimination needs to be solved before one can solve the problem of trend estimation.

Detrending refers to subtracting an estimated mean value function $\hat{m}(t)$ from a time series $X(t)$ to produce a new time series $X_{d}(t)=X(t)-\hat{m}(t)$. We call $X_{d}($.$) a detrended version of X($.$) about its mean$ value function. Detrending methods differ only in the way in which the estimated mean value function is formed. Two important ways in which one attempts to eliminate trend (or detrend) are mean detrending and linear detrending.

Mean detrending. The estimated mean value function is the arithmetic average of the time series observations:

$$
\hat{m}(t)=\frac{1}{N} \sum_{s=1}^{N} X(s)=\bar{X} \text { for } t=1,2, \ldots, N
$$

The detrended time series is

$$
X_{d}(t)=X(t)-\bar{X} ; \quad t=1,2, \ldots, N
$$

Linear detrending. The estimated mean value function is a regression line fitted to the time series by the method of least squares:

$$
\hat{m}(t)=\bar{X}+b(t-\bar{t}) \text { for } t=1,2, \ldots, N
$$

where

$$
\begin{aligned}
& \bar{X}=\frac{1}{N} \sum_{t=1}^{N} X(t), \\
& \bar{t}=\frac{1}{N} \sum_{t=1}^{N} t=(N+1) / 2, \\
& b=\frac{\sum_{t=1}^{N} t X(t)-\bar{t} \sum_{t=1}^{N} X(t)}{\sum_{t=1}^{N} t^{2}-N(\bar{t})^{2}}=\frac{\sum_{t=1}^{N} t X(t)-N \bar{t} \bar{X}}{\left(N^{2}-1\right) N / 12} .
\end{aligned}
$$

The detrended time series is

$$
X_{d}(t)=X(t)-[\bar{X}+b(t-\bar{t})], \quad t=1,2, \ldots, N .
$$

We now see why in forming sample covariances one should not necessarily subtract out the mean of a time series. We believe that one should assume 
that the time series either have zero means or represent residuals after detrending. Further, there will be occasions when automatically subtracting out the mean limits our ability to compute interesting estimates. We will often desire to consider a constant time series

$$
X(t) \equiv 1, \text { all } t
$$

which we do not desire automatically to replace by the zero time series.

\section{The Divisor Question: $N-v$ or $N$}

In the definitions given by (3.1) for $R_{i j}(v)$ we have chosen to divide by $N$ (the number of terms in the series) rather than $N-v$ (the number of terms being summed to form $R_{i j}(v)$ ). Many researchers seem to prefer to divide by $N-v$ on the grounds that it leads to an unbiased estimate of the true covariance $R_{i j}(v)$ (in the case of time series $X_{i}(\cdot)$ with zero mean):

$E\left[\frac{1}{N-v} \sum_{t=1}^{T-v} X_{i}(t) X_{j}(t+v)\right]=\frac{1}{N-v} \sum_{t=1}^{N-v} R_{i j}(v)=R_{i j}(v)$.

The suggestion that it is preferable to use the divisor $N$, rather than $N-v$, which is adopted in this work, is motivated by the following two considerations. Let us call $R_{i j}(v)$ with a divisor of $N-v$ the unbiased estimate and $R_{i j}(v)$ with a divisor of $N$ the biased estimate.

One may show that

(i) The unbiased estimate regarded as a function of $v$ is not a positive definite function while the biased estimate is. This property is desirable for two reasons; first, because we are estimating a positive definite function, and second, because it ultimately leads to nonnegative estimates of the spectral density function.

(ii) One may show that in many cases the biased estimate has a smaller mean square error than the unbiased estimate. While this seems to be true in general, a rigorous proof has not as yet been found [see Schaerf, 1963, for proofs in various special cases].

In her Stanford Ph. D. thesis, Mirella Casini Schaerf [1963] shows that while the unbiased sample covariance function usually has a greater mean square error than the biased sample covariance function, neither seems to provide a really satisfactory estimate of the true covariance function. The sample covariance functions never damp out to zero, which we assume is the case for the true covariance function. Schaerf examines various ways of modifying the sample covariance function to improve its properties, with somewhat pessimistic results.

Nevertheless it seems that when properly transformed the biased sample covariance function (in spite of failing to give a reasonable picture of the true covariance function) does yield estimates of the spectrum which provide a reasonable picture of it. It possesses the essential property which the sample covariance function must have (in order for its
Fourier transform to be interpretable as a spectral density function), namely positive definiteness.

The definition of the sample correlation function given by (3.6) is not positive definite. There seems to be no reason to use such an estimate. In what way is it relevant to our aims in time series analysis?

\section{Estimates of the Spectrum and Cross Spectrum}

The theory of estimation of the spectrum and cross spectrum is too extensive to be conveniently summarized here. We can only state the estimates which present theory seem to indicate should be formed as the first step to understanding the spectrum (I have tried to summarize this theory in Parzen [1961 and 1964]).

Three methods of computing spectra seem to be available for consideration:

(i) the indirect or transform method, which Fourier transforms weighted covariances to estimate spectra,

(ii) the direct or filter bank method, which estimates spectra as the variance and covariance of various filtered time series, (iii)

(iii) the method of autoregressive spectral estima-

We discuss method (i) in this section and method (iii) in section 8 . We do not discuss method (ii); see Ormsby [1961], Welch [1961], and Brillinger [1963].

Let $R_{i j}(v)$ denote either the sample cross covariance or the sample cross-correlation function. While in our opinion one should use the latter for ease of interpretation, the graphs one obtains have exactly the same shape in either case since they differ by constant factors (assuming (3.3) is used).

As an estimate of the true cross-spectral density function $f_{i j}(\omega)$ one forms the estimate

$$
f_{i j}(\omega)=\frac{1}{2 \pi} \sum_{|v| \leq M} e^{i v \omega} k\left(\frac{v}{M}\right) R_{i j}(v)
$$

which depend on a choice of two quantities:

(i) an integer $M$, called the truncation point of the spectral estimate (we usually choose several truncation points in practice),

(ii) a kernel $k(\cdot)$ known as the lag window of the spectral estimate; its Fourier transform

$$
K(\omega)=\frac{1}{2 \pi} \int_{-\infty}^{\infty} e^{-\mathrm{i} v \omega} k(u) d u
$$

is the spectral window generator of the estimate while

$$
K_{M}(\omega)=\frac{1}{2 \pi} \sum_{|v| \leq M} e^{\mathbf{i} v \omega} k\left(\frac{v}{M}\right)
$$

is called the spectral window of the estimate; it may be shown that approximately

$$
K_{M}(\omega)=M K(M \omega) \text {. }
$$


There is a third choice to be made in forming the estimate $f_{i j}(\omega)$, and this is the number of points on the interval 0 to $\pi$ at which it will be computed. We adopt the attitude that $f_{i j}(\omega)$ should be computed for

$$
\omega=0, \frac{\pi}{Q}, 2 \frac{\pi}{Q}, \ldots, \pi
$$

where $Q$ is an integer to be chosen. We call $Q$ the spectral computation number.

Spectral window generators. In this work, we use only the following spectral window generator:

$$
\begin{aligned}
k(u) & =1-6 u^{2}+6 u^{3}, \quad 0 \leq u \leq 0.5 \\
& =2(1-u)^{3}, \quad 0.5 \leq u \leq 1 \\
& =0, \quad u \geq 1 \\
& =k(-u), \quad u \leq 0 .
\end{aligned}
$$

A theory of spectral window generators is developed in Parzen [1964]. It is shown that the kernel (6.6) always leads to nonnegative spectral estimates whose variance (when properly normalized for comparison) is slightly less than the variance of various other estimates considered. In particular a comparison is made with certain other kernels which have been widely used; see Blackman and Tukey [1958], p. 98. One such kernel is

$$
\begin{aligned}
k(u) & =\frac{1}{2}(1+\cos \pi u), \quad|u| \leq 1 \\
& =0, \text { otherwise. }
\end{aligned}
$$

Statistical significance of spectral estimates. Assuming normality of the observed time series, it may be shown that the variance of the estimated spectral density

$$
f_{i i}^{*}(\omega)=\frac{1}{2 \pi} \sum_{|v| \leq M} e^{\mathrm{i} v \omega} k\left(\frac{v}{M}\right) R_{i i}(v)
$$

depends on the sample size $N$, the kernel $k(\cdot)$, the truncation point $M$, and the true spectral density $f_{i i}(\omega)$ as follows:

$$
\begin{aligned}
\operatorname{Var}\left[f_{i i}^{*}(\omega)\right] & =\frac{M}{N} f_{i i}^{2}(\omega)\left\{\int_{-\infty}^{\infty} k^{2}(u) d u\right\} \text { if } 0<\omega<\pi \\
& =2 \frac{M}{N} f_{i i}^{2}(\omega)\left\{\int_{-\infty}^{\infty} k^{2}(u) d u\right\} \\
& \text { if } \omega=0 \text { or } \omega=\pi .
\end{aligned}
$$

It should be noted that the equality in (6.9) is only approximate; from a rigorous point of view it should be written as a relation that holds in the limit.

Note that the variance in (6.9) increases as the truncation point $M$ increases. Therefore one is tempted to make $M$ small. However the smaller $M$ the larger is the bias of $f_{n ?}^{*}(\omega)$ as an estimate of $f_{i i}(\omega)$. Thus arises the crux of the spectral estimation problem-how to choose the truncation point so as to optimally compromise between a number of conflicting objectives.

If instead of $f_{i i}^{*}(\omega)$ one plots its logarithm $\log _{e}$ $f_{i i}^{*}(\omega)$ one achieves two advantages:

(i) one magnifies the graph of the estimated density in regions where it is small, enabling one to more easily study the behavior of the spectrum at these frequencies,

(ii) one achieves an estimate whose sampling properties are the same for all frequencies since by large sample statistical theory (6.9) implies

$$
\begin{aligned}
\operatorname{Var}\left[\log _{e} f_{i i}^{*}(\omega)\right] & =\frac{M}{N}\left\{\int_{-\infty}^{\infty} k^{2}(u) d u\right\} \text { if } 0<\omega<\pi, \\
& =2 \frac{M}{N}\left\{\int_{-\infty}^{\infty} k^{2}(u) d u\right\} \text { if } \omega=0 \text { or } \omega=\pi .
\end{aligned}
$$

Whereas a confidence band about the estimated spectral density has a width which varies with the height of the density, a confidence band about the logarithm of the density has a constant width:

$$
\left|\log _{e} f_{i i}^{*}(\omega)-\log _{e} f_{i i}(\omega)\right| \leq 2\left\{\frac{M}{N} \int_{-\infty}^{\infty} k^{2}(u) d u\right\}^{1 / 2}
$$

can be considered to be a confidence band for each $\omega$ in $0<\omega<\pi$ of approximate coniidence 95 percent.

For the kernel $(6.6), \int_{-\infty}^{\infty} k^{2}(u) d u=0.54$. Therefore the right-hand side of (6.11), hereafter denoted $\Delta$, is 0.33 for $M / N=0.05$ and 0.46 for $M / N=0.10$. The value of $\Delta$ for other ratios $M / N$ is then easily approximated (thus, $\Delta$ is $0.46 \sqrt{4}=0.92$ for $M / N=$ $0.40)$.

From (6.11) it follows that for each $\omega$ in $0<\omega<\pi$, with approximate confidence 95 percent,

$$
e^{-\Delta}-1 \leq \frac{f_{i i}^{*}(\omega)-f_{i i}(\omega)}{f_{i i}(\omega)} \leq e^{\Delta}-1 .
$$

It is worth noting how quickly the limits in (6.12) increase as $M / N$ increases; thus

\begin{tabular}{r|r|r}
\hline \hline \multicolumn{1}{c|}{$M / N$} & \multicolumn{1}{|c}{$e^{-\Delta}-1$} & $e^{\Delta}-1$ \\
\hline 0.05 & -0.3 & 0.4 \\
.10 & -.4 & .6 \\
.20 & -.5 & .9 \\
.40 & -.6 & 1.5 \\
\hline
\end{tabular}


Only for $M / N$ less than 10 percent is the percentage error of the estimated spectrum of reasonable size.

The statistical significance of cross spectral estimates is discussed by Jenkins [1963], and Goodman [1963].

Choice of truncation point. It seems to be increasingly accepted among workers in statistical spectral analysis that the spectrum should be computed for several choices of truncation point $M$. As yet we do not have routine quantitative (or even qualitative) procedures for interpreting the spectra obtained from several choices of $M$. Such procedures are still under development. My experience has led me to feel that a good picture of the spectrum can be obtained by taking three truncation points $M_{1}<M_{2}<M_{3}$. I choose $M_{1}$ to be an even number between 5 percent and 10 percent of the sample size $N$. I then choose $M_{2}=2 M_{1}$ and $M_{3}=2 M_{2}$. In section 9 we give examples of spectra computed with several truncation points.

Choice of spectral computation number. The spectral computation point $Q$ has in the past frequently been chosen to be equal to the truncation point $\dot{M}$. One can prove a sampling theorem to the effect that the estimated spectrum (which is a function of $\omega$, measured in cycles per unit of observation time, in the interval $0 \leq \omega \leq 0.5)$ can be recovered from its value at $M$ equally spaced points. However, this recovery cannot necessarily be done by linear interpolation. If the graph of the estimated spectrum is to be obtained by merely drawing line segments connecting the computed values, one needs to compute the spectrum at $Q$ equispaced frequencies, where $Q$ should be at least $2 M$ and perhaps should be $4 M$.

If one uses 3 truncation points $M_{1}<M_{2}<M_{3}$, it has seemed reasonable to me to compute each spectrum at $Q=M_{3}$ points. However, one should choose $Q$ (approximately equal to $M_{3}$ ) such that the frequencies which are multiples of $\pi / Q$ are of physical interest. For economic time series of monthly data we usually choose $Q$ to be a multiple of 12 .

\section{Computation Formulas for Cross-Spectral Estimates by the Transform Method}

The time series $X_{1}(\cdot)$ and $X_{2}(\cdot)$ whose cross spectra one is estimating are usually not the directly observed time series but rather the result of various detrending and filtering operations. Assuming that we are dealing with two time series ready for cross-spectral analysis, the following computations are performed.

STEP 1. Let $N$ be the number of observations in the two series. Choose an integer $V_{\max }<N$ and compute, for $v=0,1,2, \ldots, V_{\max }$, the cross covariances

$$
R_{11}(v), R_{22}(v), R_{12}(v), R_{21}(v)
$$

and the cross correlations which we denote by the notation

$$
\bar{R}_{11}(v), \bar{R}_{22}(v), \bar{R}_{12}(v), \bar{R}_{21}(v) .
$$

Step 2. Choose three truncation points $M_{1}, M_{2}$, $M_{3}$. For each truncation point, carry out steps 3 to 5 .

Step 3. Choose an integer Q. Then for each frequency

$$
\omega=0, \frac{\pi}{Q}, 2 \frac{\pi}{Q}, \ldots, \pi
$$

compute the quantities described in steps 4 and 5 .

Step 4. We prefer to compute normalized cross spectra (the transforms of cross correlations rather than of cross covariances) since they seem to be easier to graph and to compare.

The normalized spectral density functions, cospectrum, and quadrature spectrum

$$
f_{11}(\omega), f_{22}(\omega), c_{12}(\omega), q_{12}(\omega)
$$

at frequency $\omega$ could be computed by the formulas

$$
\begin{gathered}
f_{i i}(\omega)=\frac{1}{\pi}\left\{\frac{1}{2} \bar{R}_{12}(0)+\sum_{v=1}^{M} \cos (v \omega) k\left(\frac{v}{M}\right) \bar{R}_{i i}(v)\right\}, \\
c_{12}(\omega)=\frac{1}{\pi}\left\{\frac{1}{2} \bar{R}_{12}(0)+\frac{1}{2} \sum_{v=1}^{M} \cos (v \omega) k\left(\frac{v}{M}\right)\right. \\
{\left[\bar{R}_{12}(v)+\bar{R}_{21}(v)\right]} \\
q_{12}(\omega)=\frac{1}{2 \pi} \sum_{v=1}^{M} \sin (v \omega) k\left(\frac{v}{M}\right)\left[\bar{R}_{12}(v)-\bar{R}_{21}(v)\right]
\end{gathered}
$$

where $M$ denotes the truncation point.

These spectral quantities are most efficiently computed not by using the explicit formulas above but by using an efficient procedure for evaluation of finite Fourier transforms due to Goertzel [1960].

Step 5. We next compute the following spectral quantities:

Amplitude, $\quad A_{12}(\omega)=\left|f_{12}(\omega)\right|=\left\{\left|c_{12}(\omega)\right|^{2}+\left|q_{12}(\omega)\right|^{2}\right\}^{1 / 2}$

Phase,

$$
\varphi_{12}(\omega)=\tan ^{-1}\left\{q_{12}(\omega) / c_{12}(\omega)\right\}
$$

Coherence, $\quad W_{12}(\omega)=\left|f_{12}(\omega)\right|^{2} \div f_{11}(\omega) f_{22}(\omega)$

Gain $1 / 2, \quad G_{1 / 2}(\omega)=A_{12}(\omega) \div f_{22}(\omega)$

Gain $2 / 1, \quad G_{2 / 1}(\omega)=A_{12}(\omega) \div f_{11}(\omega)$.

Computation time. It may be worth explicitly noting two ways which we have found for reducing the computation time required.

In computing covariances we call upon an external procedure for computing inner products which executes faster than the equivalent coding in programming language. Figure 7 a lists our covariance computation routine.

In computing spectra and cross spectra we use the Goertzel procedure mentioned after (7.7). Figure $7 \mathrm{~b}$ lists this computation routine. 


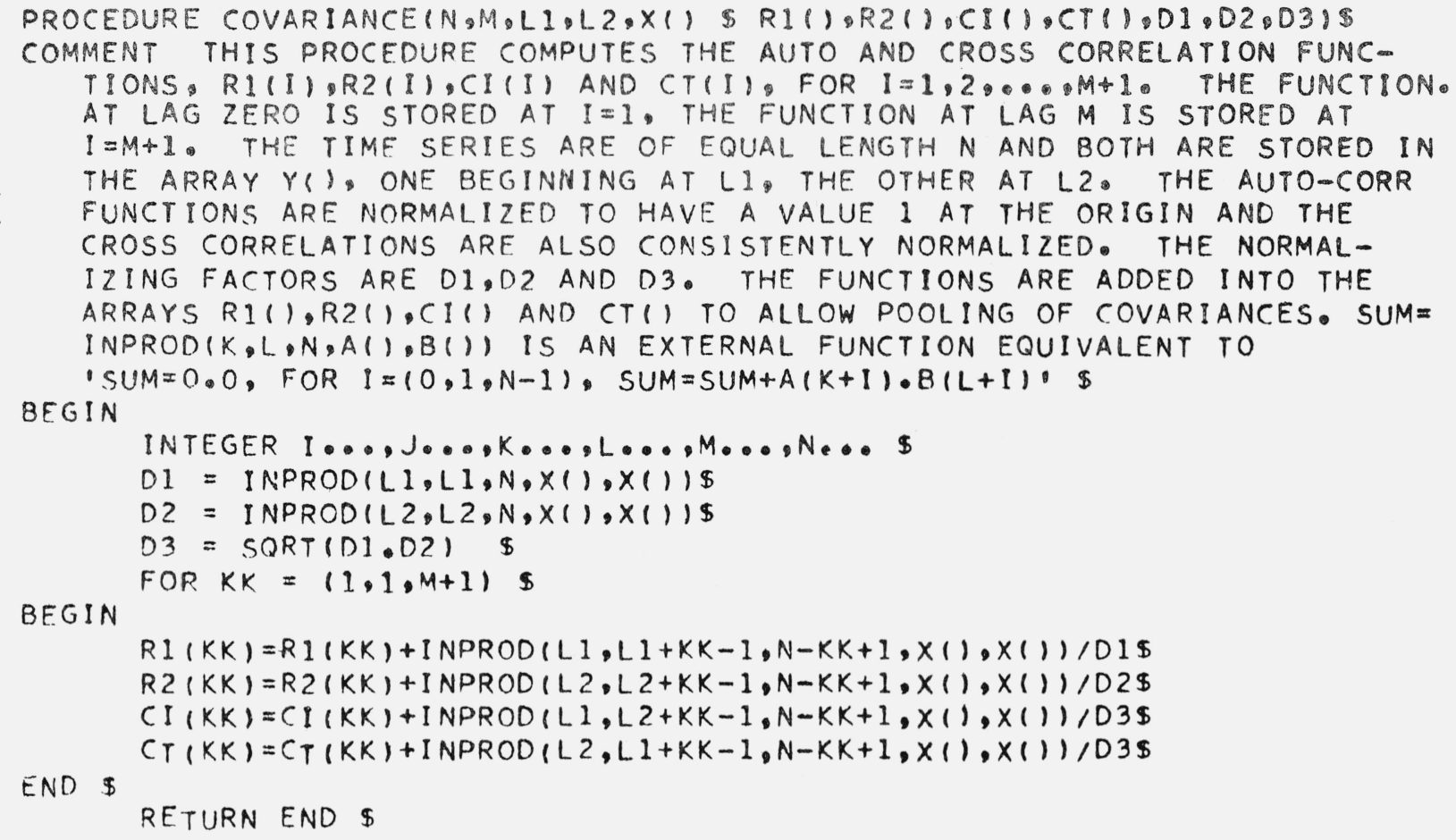

Figure 7a. Procedure covariance.

The computation time $T$ of the covariances of a pair of time series is approximately the product of $N$ (the length of the time series) and $M$ (the truncation point). Some typical values (on a 7090) are:

\begin{tabular}{r|r|r}
\hline \multicolumn{1}{|r|}{$N$} & \multicolumn{1}{|r|}{$T$} \\
\hline 200 & 80 & 4 sec. \\
1000 & 200 & $1 \mathrm{~min}$. \\
4000 & 500 & $8 \mathrm{~min}$. \\
\hline
\end{tabular}

The computation time $T$ of a cross-spectral analysis is approximately the product of $M$ (the truncation point) and $Q$ (the spectrum computation number). Some typical values (on a 7090) are:

\begin{tabular}{|c|c|c|}
\hline$M$ & $Q$ & $T$ \\
\hline $\begin{array}{r}40 \\
80 \\
80 \\
200 \\
500\end{array}$ & $\begin{array}{r}40 \\
80 \\
160 \\
200 \\
200\end{array}$ & $\begin{array}{l}1 \text { sec. } \\
4 \text { sec. } \\
8 \text { sec. } \\
24 \text { sec. } \\
1 \text { min. }\end{array}$ \\
\hline
\end{tabular}

\section{Autoregressive Spectral Estimation}

Given autoregression coefficients $a_{1}, \ldots, a_{m}$ one can form a new time series, denoted $\hat{X}(\cdot)$, from a given time series $X(\cdot)$, by the formula

$$
\hat{X}(t)=a_{1} X(t-1)+\ldots+a_{m} X(t-m) .
$$

We say that $\hat{X}(\cdot)$ is obtained by autoregressive filtering from $X(\cdot)$. The residuals

$$
\epsilon(t)=X(t)-\hat{X}(t)
$$

are said to be autoregressive residuals. They are examined to determine how good a predictor $\hat{X}(t)$ is of $X(t)$.

The autoregressive coefficients $a_{1}, \ldots, a_{m}$ may either be specified a priori or may be estimated from the data by a procedure known as stagewise autoregressive estimation (see fig. 8a for the computation routine). In this case, one may be able to estimate the unknown spectral density function of the time series $X(\cdot)$ by a method called autoregressive spectral estimation. To describe this method we discuss some relations between transfer functions. 


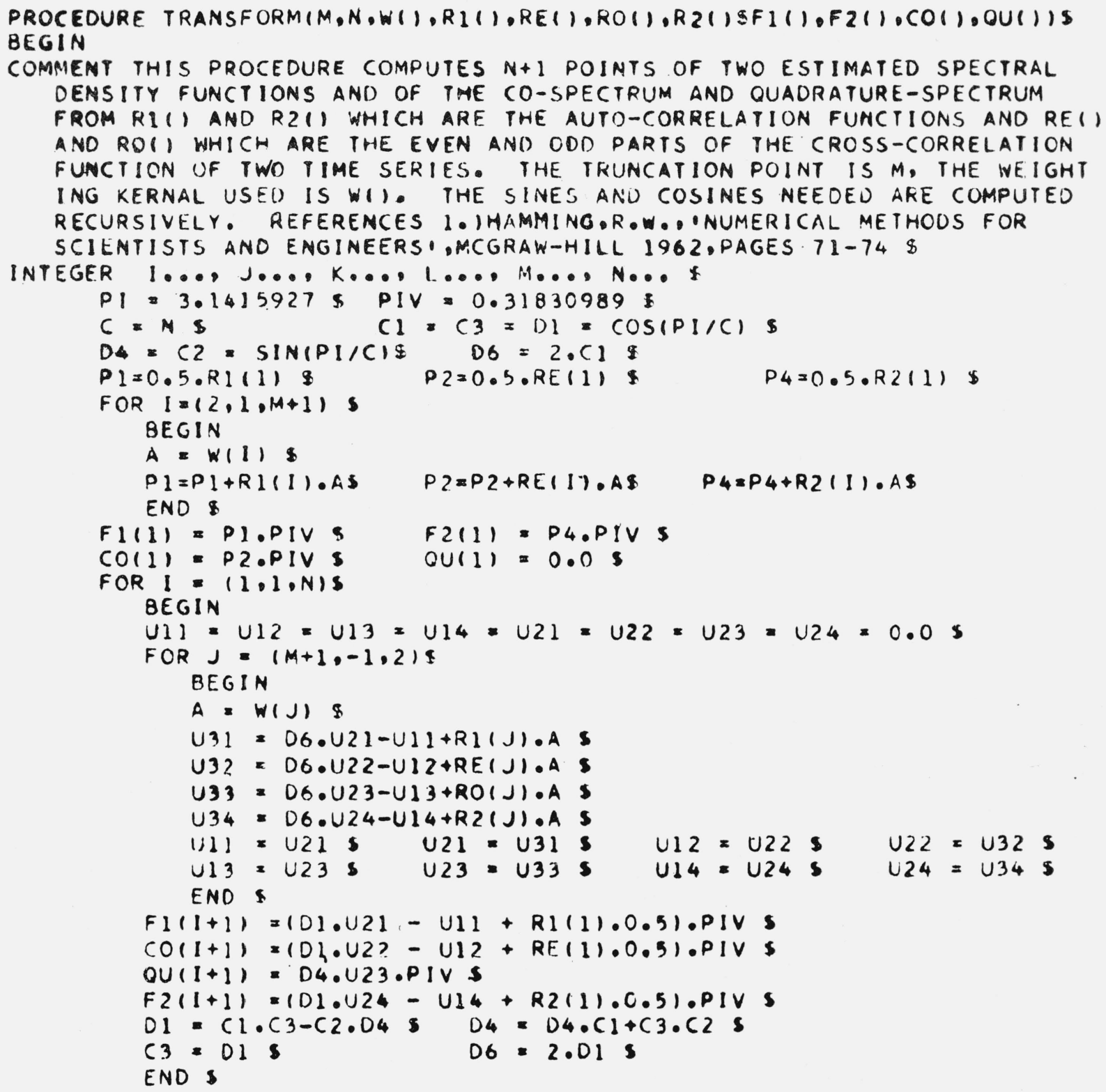

Figure 7b. Procedure transform.

If the given time series $X(\cdot)$ actually was a white noise process, with spectral density function

$$
f_{X}(\omega)=\frac{1}{2 \pi}, \quad|\omega| \leq 2 \pi
$$

then the time series $\epsilon(\cdot)$, defined by (8.2) would have as its spectral density

$$
f_{\epsilon}(\omega)=f_{X}(\omega) \cdot|A(\omega)|^{2}
$$

where $A(\omega)$ is the frequency transfer function

$$
A(\omega)=1-a_{1} e^{-i \omega}-\ldots-a_{m} e^{-m i \omega},
$$

representing (up to a factor $e^{i \omega t}$ ) the output of the operation defining $\epsilon($.$) when the input is e^{i \omega t}$. Therefore

$$
\begin{gathered}
f_{\epsilon}(\omega)=\frac{1}{2 \pi}\left\{\left(1-a_{1} \cos \omega-\ldots-a_{m} \cos m \omega\right)^{2}\right. \\
\left.+\left(a_{1} \sin \omega+\ldots+a_{m} \sin m \omega\right)^{2}\right\} .
\end{gathered}
$$

Given autoregressive coefficients $a_{1}, \ldots, a_{m}$ we denote the right-hand side of (8.6) by TRW $(\omega)$, and call it the white noise transfer function of the scheme.

We desire to normalize the white noise transfer function to have unit area (over the interval $|\omega| \leq \pi$ ). 


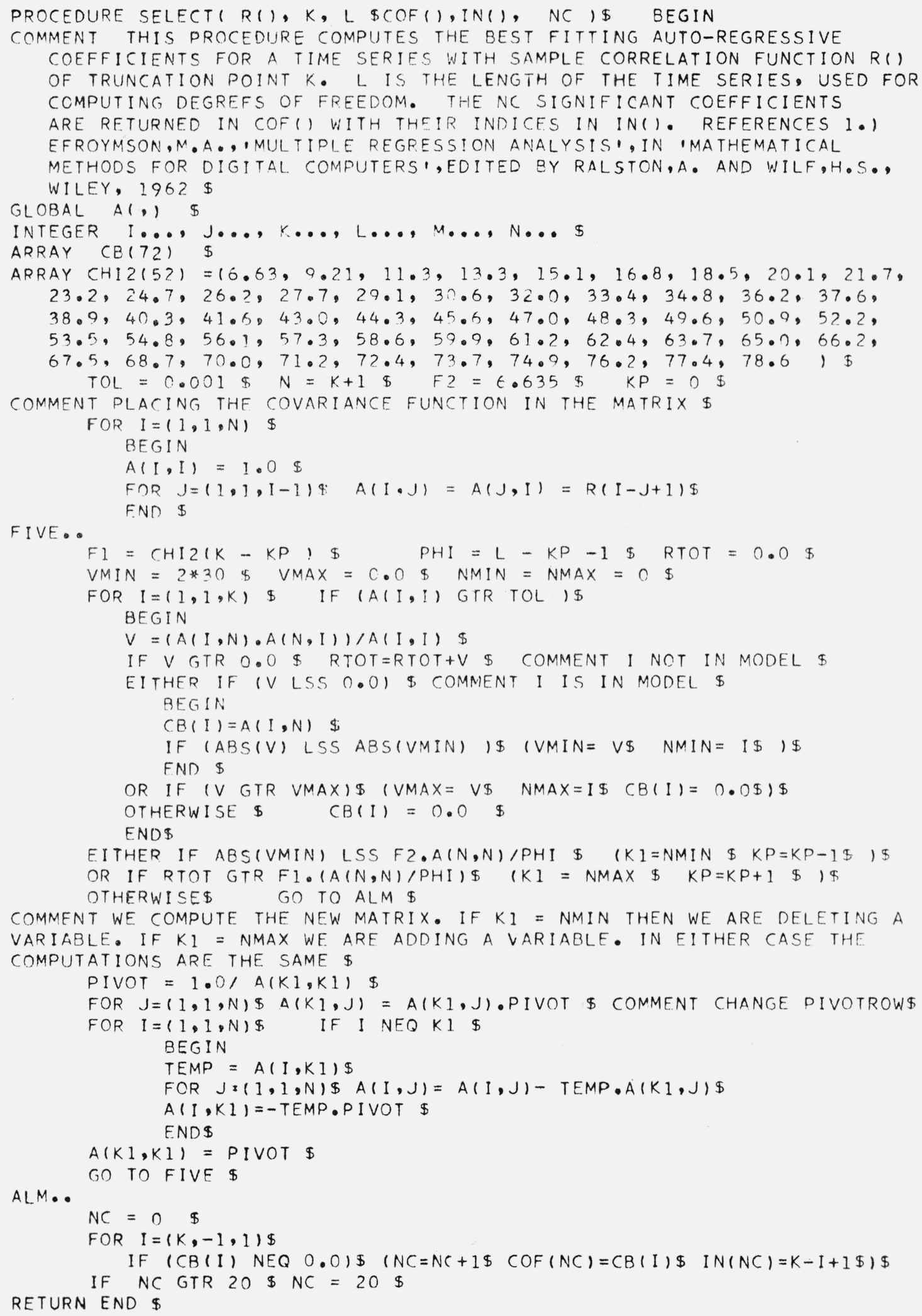

Figure 8a. Procedure select. 
One may verify that

$$
\int_{-\pi}^{\pi} \operatorname{TRW}(\omega) d \omega=1+a_{1}^{2}+\ldots+a_{m}^{2} .
$$

Therefore the normalized white noise transfer function, denoted by TRWN $(\omega)$, is given by

$$
\operatorname{TRWN}(\omega)=\frac{\operatorname{TRW}(\omega)}{1+a_{1}^{2}+\ldots+a_{m}^{2}} .
$$

Note that this transfer function is normalized to have unit area over the interval $-\pi \leq \omega \leq \pi$.

If $X(\cdot)$ is in fact an autoregressive scheme satisfying the model

$$
X(t)=a_{1} X(t-1)+\ldots+a_{m} X(t-m)+\epsilon(t),
$$

where $\epsilon(\cdot)$ is white noise, then from (8.4) its spectral density function is given by

$$
\begin{aligned}
f_{X}(\omega)= & f_{\epsilon}(u) \div|A(\omega)|^{2} \\
=[2 \pi & \left\{\left(1-a_{1} \cos \omega-\ldots-a_{m} \cos m \omega\right)^{2}\right. \\
& \left.\left.+\left(a_{1} \sin \omega+\ldots+a_{m} \sin m \omega\right)^{2}\right\}\right]^{-1} .
\end{aligned}
$$

We denote the right-hand side of (8.10) by TRAR $(\omega)$, and call it the autoregressive transfer function of the scheme of coefficients $a_{1}, \ldots$.., $a_{m}$. Note that

$$
\operatorname{TRAR}(\omega)=\{4 \pi \operatorname{TRW}(\omega)\}^{-1} .
$$

We have not been able as yet to find a formula (convenient for computation) for the integral $\int_{-\pi}^{\pi} \operatorname{TRAR}(\omega) d \omega . \quad$ Since we prefer to compute normalized transfer functions, in practice we approximately evaluate this integral by a crude method of numerical integration and thus compute the normalized autoregressive transfer function, denoted by TRARN $(\omega)$ and given by

$$
\operatorname{TRARN}(\omega)=\frac{\{4 \pi \operatorname{TRW}(\omega)\}^{-1}}{\int_{-\pi}^{\pi}\{4 \pi \operatorname{TRW}(\omega)\}^{-1} d \omega} .
$$

We can describe the method of autoregressive spectral estimation. It consists of (i) determining autoregressive coefficients $a_{1}, \ldots a_{m}$ by stagewise autoregressive estimation, (ii) estimating the spectral density function $f_{\epsilon}(\omega)$ of the autoregressive residuals, (iii) if $f_{\epsilon}(\omega)$ is approximately the spectrum of white noise, we take the normalized autoregressive transfer function, deîned by (8.12), as the estimated spectrum of the original time series $X(t)$.

The statistical theory of stagewise autoregressive model fitting is considered by Schaerf [1963].

\section{Some Examples of Empirical Time Series Analysis}

One of the major aims of empirical time series analysis is to provide a modern solution to what classically has been called "the search for hidden periodicities." Classical approaches to this problem have been discredited because they seemed to provide evidence for the existence of "spurious cycles." One way to avoid seeing cycles in data where they are not present is to compare estimated spectra from this data with the estimates formed under the same sample size and truncation point from data of known properties.

As an example of the above method, an analysis of an economic time series will be carried through. The radio case would be essentially the same, apart perhaps from changes in length and time scale in the series, and the economic material has already been analyzed by our method.

Given a series of length 180 (as many economic time series are) one might choose a minimum truncation point of 16 ; the other truncation points would then be 32 and 64 .

To obtain some idea of the resolution of our spectral windows, it is instructive to first perform a spectral analysis of a constant time series

$$
X_{1}(t)=1, \quad t=1,2, \ldots, N .
$$

No mean subtraction is performed. The sample covariance function is given by

$$
R(v)=\frac{1}{T} \sum_{t=1}^{T-v} X(t) X(t+v)=1-\frac{v}{T} .
$$

The resulting estimated spectral density functions are given in figure $9 \mathrm{a}$. The curves corresponding to different truncation points are easily distinguished since at zero frequency the curves increase in amplitude with increasing truncation point. The spectral density function corresponding to $M=16$ has a local maximum of 0.175 which might be construed as a "spurious" cycle.

Series of stock price indexes have been extensively analyzed (see Granger and Morgenstern [1963]). We consider a monthly series of such an index, denoted $X_{2}(t)$, for the 180 months in 1948-1962, taking logarithms and mean detrending. The estimated spectra, given in figure $9 \mathrm{~b}$, are exactly as in figure $9 \mathrm{a}$, except for a little additional power at high frequencies.

Similarly we estimated the spectra of the linear series

$$
X_{3}(t)=t, \quad t=1,2, \ldots, 180
$$

with no detrending. The estimated spectra were again exactly as in figure 9 a.

The conclusion to be drawn is that for both the stock price index series and the linear series, the spectrum consists essentially of a single line (or spike) at zero frequency; a more precise statement of this assertion might perhaps be made using techniques of mixed spectral analysis such as those currently being investigated by George Hext [1964].

To characterize more clearly the mechanism generating the stock price index series and the linear series, other than to say they have a spectrum concentrated at zero frequency, one fits these series by autoregressive schemes using stagewise autoregressive estimation. For the stock price index series one finds the scheme

$$
X_{2}(t)=0.986 X_{2}(t-1)+\epsilon_{2}(t)
$$




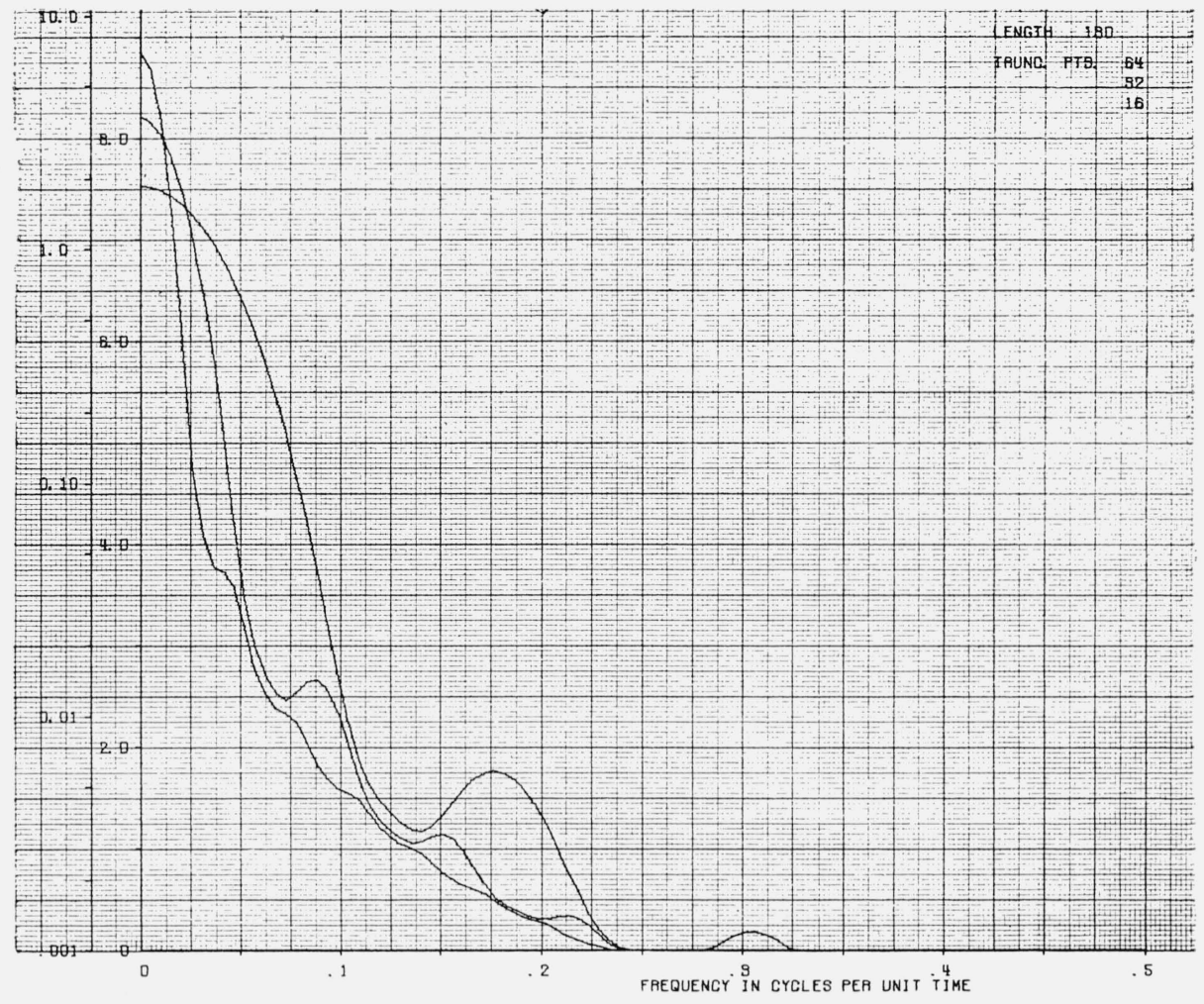

Figure 9a. Log of estimated spectral density functions, constant series.

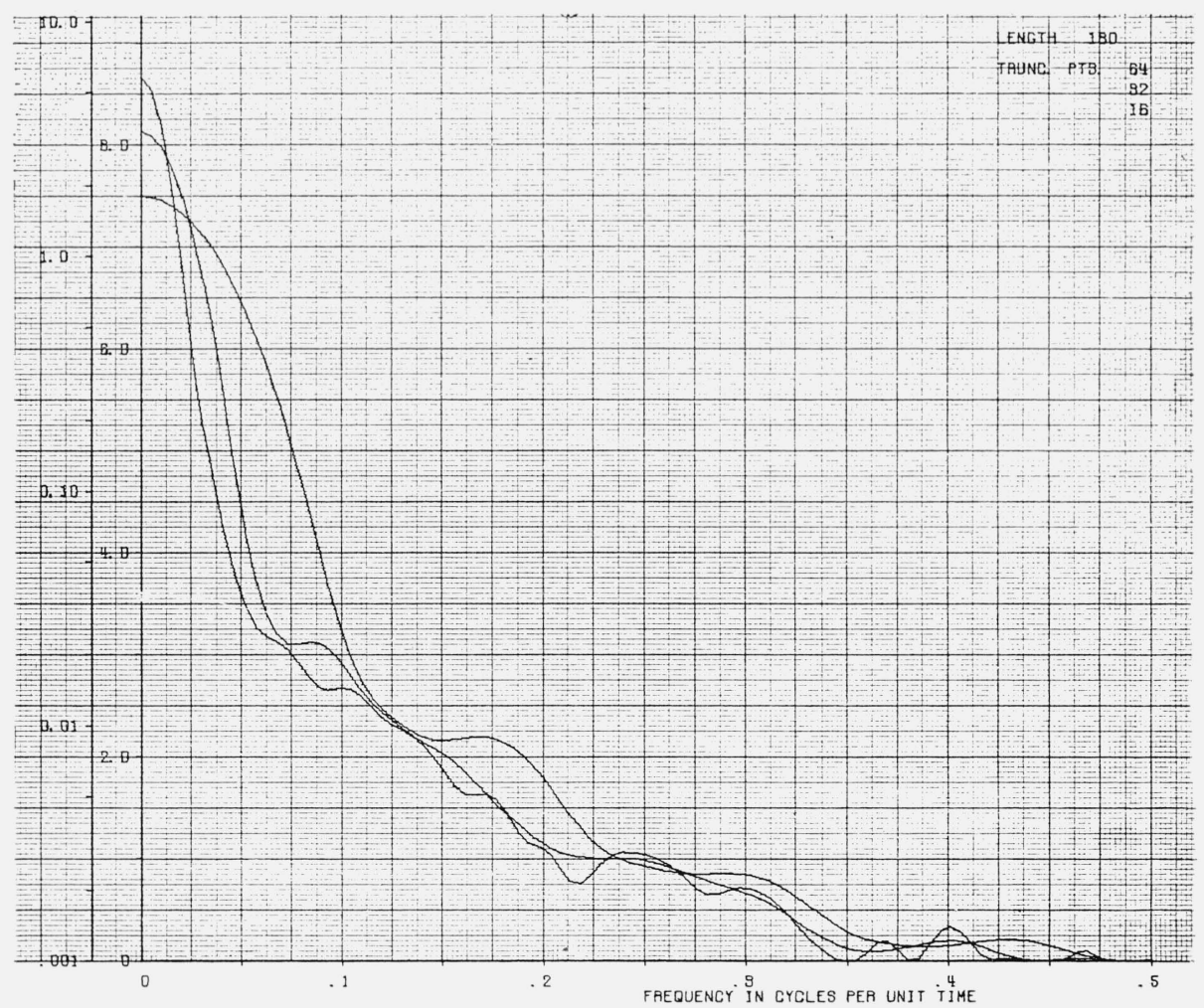

FiguRE 9b. Log of estimated spectral density functions, stock price index 48-62, mean detrend. 


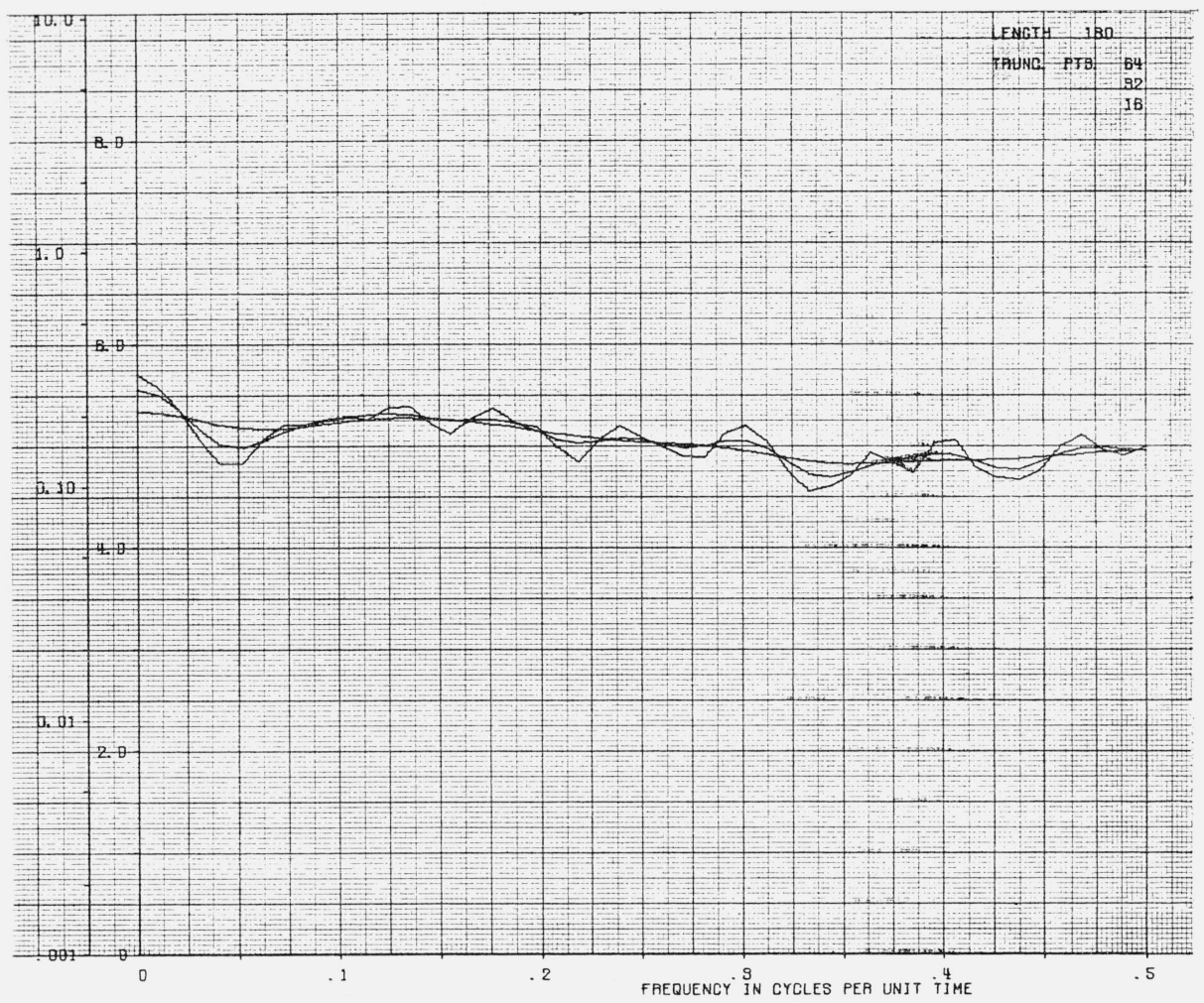

FIGURE 9c. Log of estimated spectral density functions, stock price stageauto.

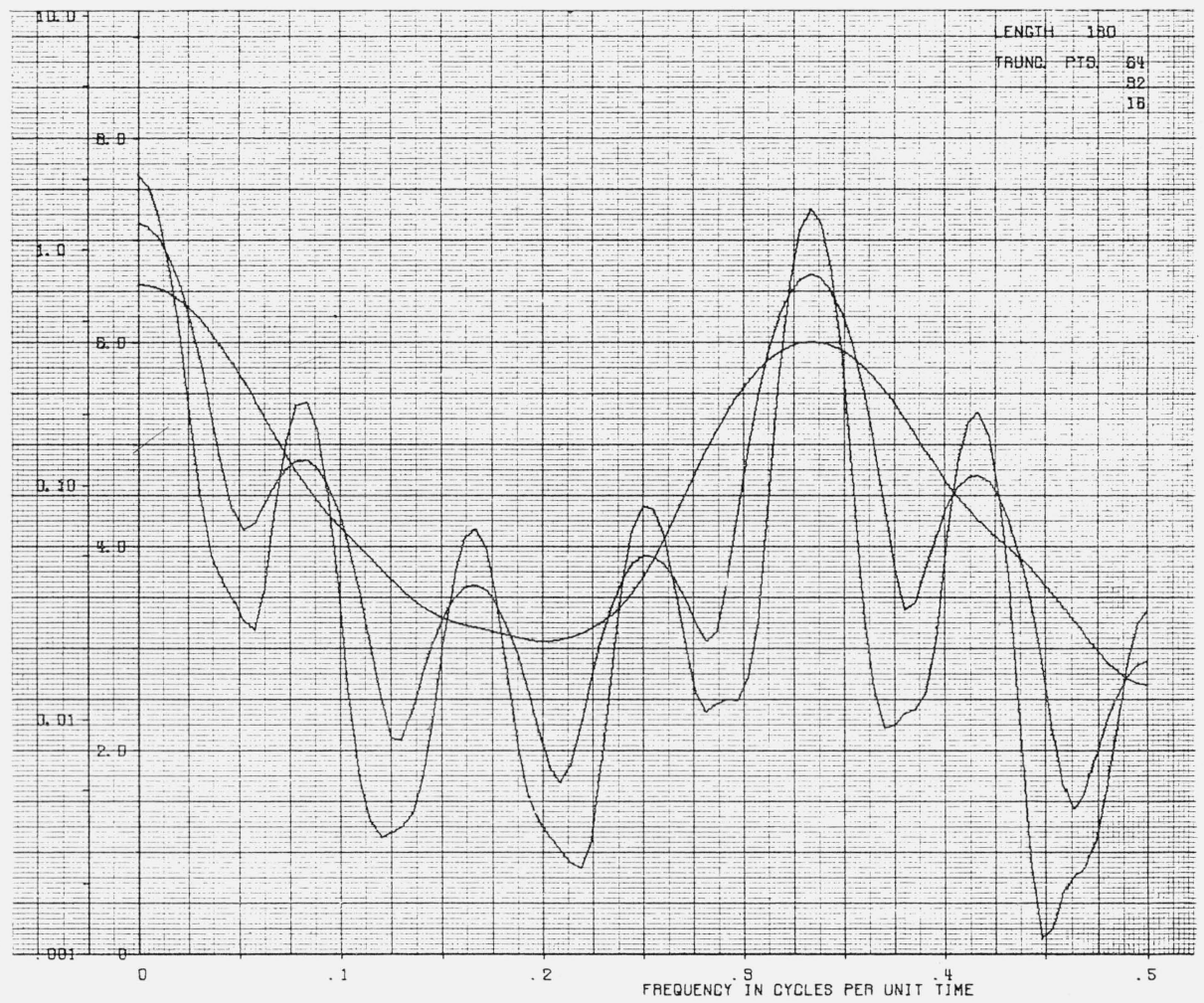

Figure 9d. Log of estimated spectral density functions, Fed. cash from public 48-62 mean detrend. 


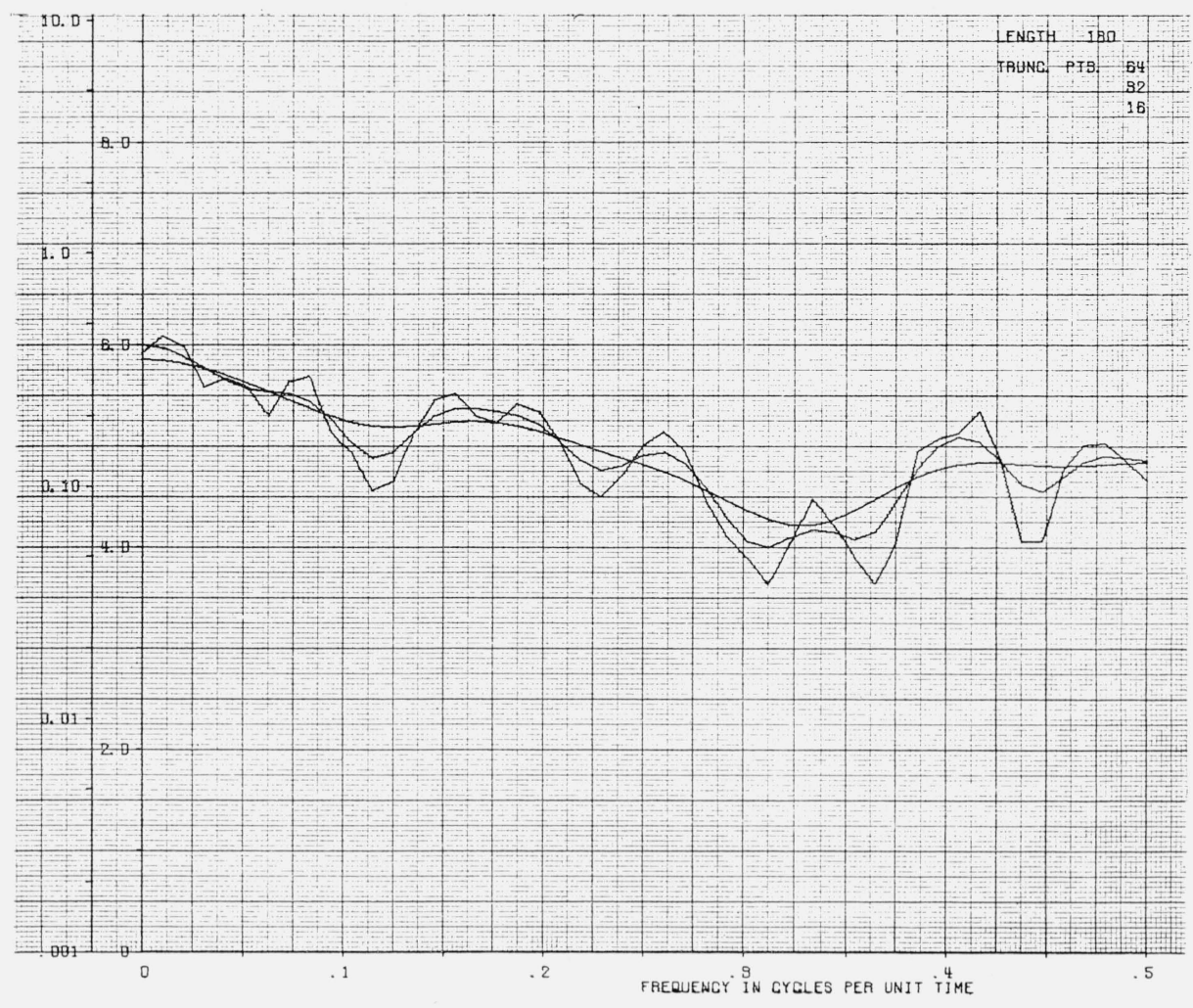

Figure 9e. Log of estimated spectral density functions, Fed. cash stageauto.

while for the linear series

$$
X_{3}(t)=0.992 X_{3}(t-1)+\epsilon_{3}(t) .
$$

We then estimate the spectra of the residual time series $\epsilon_{2}(t)$ and $\epsilon_{3}(t)$.

The estimated spectral density functions of the stock price index residuals are given in figure $9 \mathrm{c}$; they are well within the confidence limits of white noise spectra. The true spectrum of white noise on the logarithmic plot used in the figure is a horizontal line at

$$
\log _{e}\left(1000 \frac{1}{2 \pi}\right)=5.07
$$

From the foregoing anaiysis we have essentially obtained the often found random walk model for the logarithms of stock market prices $X_{2}(t)$ :

$$
X_{2}(t)=X_{2}(t-1)+\epsilon_{2}(t)
$$

where $\boldsymbol{\epsilon}_{2}(t)$ is a white noise series. It would be interesting to investigate whether the models (9.4) and (9.7) are significantly different.

The estimated spectral density functions of the linear series residuals are again exactly as in figure 9a. Examination of the printed residuals $\epsilon_{3}(t)$ shows that they are given by a linear series

$$
X_{3}(t)=1+(t-1) 0.00831, \quad t=1,2, \ldots
$$

Performing a stagewise autoregression on this series disclosed that it approximately satisfies the model $\epsilon_{3}(t)=0.994 \epsilon_{3}(t-1)$; one would conclude that the original series $X_{3}(t)$ satisfies $X_{3}(t)-2 X_{3}(t-1)+X_{3}(t-2)=0$ and thus is a straight line.
Our last example is intended to illustrate how autore gressive spectral estimation is used to check the results of the transform method of spectral estimation. Let $X_{4}(t)$ denote a monthly series of logarithms of cash payments received by the Federal Government from the public. Its spectra are graphed in figure $9 \mathrm{~d}$. There are prominent peaks at $\omega=0$ and $\omega=0.33$. There are questionable peaks at $0.083,0.167,0.25,0.42$ (for the interpretation of these frequencies in terms of periods see the table below).

Fitting an autoregressive scheme to $X_{4}(t)$ by stagewise autoregressive estimation one finds the scheme

$$
\begin{aligned}
X_{4}(t)=0.732 X_{4}(t-3)+0.825 X_{4}(t-12) & -0.611 X_{4}(t-15)+\epsilon_{4}(t) .
\end{aligned}
$$

The spectra of the residuals $\epsilon_{4}(t)$, given in figure $9 \mathrm{e}$, are not quite white; there is a slight predominance of power at low frequency which should be investigated. Nevertheless, it is white enough for us to feel justified in taking the normalized autoregressive transfer function as an estimate of the spectrum of $X_{4}(t)$. This transfer function is given in the $1 /$ TRANSFER column of figure 9f. All the peaks previously found are still present, with their relative size clearly indicated. The model given by (9.9) lends itself readily to prediction. The relative mean square prediction error is defined to be

$$
\frac{\operatorname{Var}\left[\epsilon_{4}(t)\right]}{\operatorname{Var}\left[X_{4}(t)\right]}
$$

These variances are routinely computed in our program. Their ratio turns out to be about 0.1 ; the predictor is thus rather reliable.

Associating frequencies and periods. Consider a time series observed at monthly intervals. The following table shows the frequencies corresponding to several important periods. 
$.7322 \times(1-3)$

$.8252 \times(T-12)$

$-.6103 \times(T-15)$

FREQ.

TRANSFER

.0002

.0030

.0185

.0553

.1025

.1271

.1018

.0401

.0028

.0571

.2165

.4123

.5285

.4841

.2998

.0941

.0054

.0941

.2998

.4841

.5285

.4123

.2165

.0571

.0028

.0401

.1018

.1271

.1025

.0553

.0185

.0030

.0002

.0030

.0185

.0553

.1025

.1271

.1018

.0401

.0028

.0571

.2165

.4123

.5285

.4841

.2998

.0941

.0054

\section{1/TRANSFER}

3. 8218

.2214

- C 362

.0121

.0065

.0053

.0066

.0167

.2403

.0117

.0031

.0016

.0013

.0014

.0022

.0071

.1241

.0071

.0022

.0014

.0013

.0016

.0031

.0117

.2403

.0167

. 0066

.0053

.0065

.0121

.0362

.2214

3. 8218

.2214

.0362

.0121

.0065

.0053

.0066

.0167

.2403

.0117

.0031

.0016

.0013

.0014

.0022

.0071

.1241
LOC (TRANS)

$-8.6503$

$-5.3020$

$-3.9906$

$-2.8949$

$-2.2777$

$-2.0625$

$-2.2851$

$-3.2163$

$-5.8840$

$-2.8636$

$-1.5299$

$-.8860$

$-.6377$

$-.7254$

$-1.2046$

$-2.3629$

$-5.2228$

$-2.3629$

$-1.2046$

$-.7254$

$-.6377$

$-.8860$

$-1.5299$

$-2.8636$

$-5.8840$

$-3.2163$

$-2.2851$

$-2.0625$

$-2.2777$

$-2.8949$

$-3.9906$

$-5.8020$

$-8.6503$

$-5.8020$

$-3.9906$

$-2.8949$

$-2.2777$

$-2.0625$

$-2.2851$

$-3.2163$

$-5.8839$

$-2.8636$

$-1.5299$

$-.8860$

$-.6377$

$-.7254$

$-1.2046$

$-2.3629$

$-5.2228$
LOG ( $1 /$ TRANS )

1.3407

$-1.5076$

$-3.3190$

$-4.4147$

$-5.0319$

$-5.2471$

$-5.0245$

$-4.0933$

$-1.4257$

$-4.4461$

$-5.7797$

$-6.4236$

$-6.6720$

$-6.5843$

$-6.1050$

$-4.9467$

$-2.0869$

$-4.9467$

$-6.1050$

$-6.5843$

$-6.6720$

$-6.4236$

$-5.7797$

$-4.4461$

$-1.4257$

$-4.0933$

$-5.0245$

$-5.2471$

$-5.0319$

$-4.4147$

$-3.3190$

$-1.5076$

1. 3407

$-1.5077$

$-3.3190$

$-4.4147$

$-5.0319$

$-5.2471$

$-5.0245$

$-4.0933$

$-1.4257$

$-4.4461$

$-5.7797$

$-6.4236$

$-6.6720$

$-6.5843$

$-6.1050$

$-4.9467$

$-2.0869$

Figure 9f. Transfer functions. 


\begin{tabular}{c|c}
\hline $\begin{array}{c}\text { Period } \theta \text {, length } \\
\text { of a cycle }\end{array}$ & $\begin{array}{c}\text { Frequency }=1 / \theta \\
\text { cycles per month }\end{array}$ \\
\hline & 0.000 \\
2 & .500 \\
3 & .333 \\
4 & .250 \\
6 & .167 \\
12 & .083 \\
24 & .042 \\
48 & .021 \\
\hline
\end{tabular}

It is a pleasure to thank Howard Taylor who contributed the bulk of the computer program that we use for empirical time series analysis. I desire to express my appreciation for the great intelligence, ingenuity, persistence, and patience that he brought to bear on this work.

\section{References}

Blackman, R. B., and J. W. Tukey (1958), The Measurement of Power Spectra from the Point of View of Communications Engineering, p. 98 (Dover Publications, New York, N.Y.).

Brillinger, D. R. (1963), The design of digital filters for discrete time series, 34th Meeting, Ottawa in 1963 (To be published in the Bulletin of International Statistical Institute).

Goertzel, G. (1960), Fourier analysis, Mathematical Methods for Digital Computers, ed. A. Ralston and H. S. Wilf, ch. 24, pp. 263-279 (John Wiley \& Sons, Inc., New York, N.Y.).

Goodman, N. R. (1963), Spectral analysis of multiple stationary time series, Proc. Symposium on Time Series Analysis, ed. Murray Rosenblatt, pp. 260-266 (John Wiley \& Sons, Inc., New York, N.Y.).

Granger, C. W. J., and O. Morgenstern (1963), Spectral analysis of New York stock market prices, Kyklos 16, 1-27.

Hamming, R. W. (1962), Numerical Methods for Scientists and Engineers (McGraw-Hill Book Co., Inc., New York, N.Y.).

Hext, G. (1964), A new approach to time series with mixed spectra, Stanford Ph. D. thesis, Stanford University, Stanford, Calif.

Jenkins, G. M. (1963), Cross-spectral analysis and the estimation of linear open loop transfer functions, Proc. Symposium on Time Series Analysis, ed. Murray Rosenblatt, pp. 267-278 (John Wiley \& Sons, Inc., New York, N.Y.).

Ormsby, J. (1961), Design of numerical filters with applications to missile data processing, J. Assoc. Comp. Mach. 8, $440-466$.

Parzen, E. (1961), Mathematical considerations in the estimation of spectra, Technometries 3, 167-190.

Parzen, E. (1962), Stochastic Processes (Holden-Day, Inc., San Francisco, Calif.).

Parzen, E. (1964), On statistical spectral analysis. To be published by the American Mathematical Society in the Proceedings of a Symposium on Stochastic Processes in Mathematical Physics and Engineering.

Schaerf, M. C., (1963), Estimation of the covariance and autoregressive structure of a stationary time series, Stanford $\mathrm{Ph}$. D. thesis.

Welch, P. D., (1961), A direct digital method of power spectrum estimation, IBM Journal of Research and Development, 5, 141-156. 